\title{
In Memoriam Kristofer M. Schipper (1934-2021)
}

\author{
Vincent Goossaert \\ École Pratique des Hautes Études (EPHE, PSL)
}

An English version of this obituary is freely available at https://brill.com/file asset/downloads_products/7547_tpao_inmemoriam_schipper.pdf.

Kristofer M. Schipper, connu de ses collègues, amis et élèves sous le nom de Rik, s' est éteint le 18 février 2021 dans un hôpital d'Amsterdam, victime d'un brutal accident vasculaire. Il laisse une immense œuvre scientifique et il a formé ou profondément influencé des dizaines de chercheurs qui enseignent aujourd'hui dans des universités du monde entier, en Asie, en Europe et aux États-Unis'.

Rik est né le 23 octobre 1934 à Jarnskog (Suède) et a grandi en Hollande, dans un village près d'Amsterdam où son père adoptif Klaas Abe Schipper (1906-1949), un intellectuel progressiste aux sympathies socialistes, était pasteur de l'Église mennonite néerlandaise. Sa mère, Johanna Engelberta Kuiper (1896-1956), était une brillante femme de lettres, dont les romans pour jeunes lecteurs sont encore lus aujourd'hui. Leurs convictions les amenèrent à recueillir bon nombre de juifs, dont des enfants, pendant l'occupation nazie; ils furent dénoncés à la Gestapo et son père, capturé et déporté; il ne survivra que quelques années après la Libération. Sa mère parvint à s' enfuir avec Rik, son frère et plusieurs enfants recueillis; ils vécurent de longs mois cachés dans une maison à Amsterdam². Il passa la fin de la guerre en internat, mais garda un lien indéfectible avec ses compagnons de fuite, ainsi qu'une haine viscérale pour les idéologies nationalistes et totalitaires. Il demandera et obtiendra pour ses parents la reconnaissance de «Justes parmi les nations»; désormais, un monument les honore dans le village de son enfance.

En 1955, sa mère démissionne du poste de pasteure qu' elle occupe dans l'Eglise mennonite d'Amsterdam et part en Indonésie où vivent son frère et

1 Je suis profondément reconnaissant à Caroline Gyss, Fang Ling et Isabelle Ang qui ont relu ce texte et l'ont amélioré en de très nombreux points.

2 Kristofer Schipper 施舟人, Tongnian 童年. L'enfance, tr. Yuan Bingling 袁冰凌 (Beijing, Beijing daxue chubanshe, 2005), 93-200. 
son fils aîné; elle y décédera en 1956 sans que Rik ait eu l'occasion de la revoir. Après son baccalauréat au Lycée (gymnasium) Montessori d'Amsterdam en 1954, Rik décide de se consacrer aux arts et aux humanités et quitte les Pays-Bas pour venir à Paris. Il pense d' abord se tourner vers le marché de l' art et étudie à l'école du Louvre, où il découvre l' art chinois, qui le fascine, et dont il est diplômé en 1958. Il étudie simultanément les langues et est diplômé de chinois à l'École nationale des langues orientales vivantes en 1958, puis de japonais en 1960. Ayant désormais accès aux textes comme aux images, il entreprend l'étude des visions du monde ayant produit les chefs-d'œuvre de la peinture et des autres arts chinois. En Sorbonne, à la Section des Sciences religieuses de l'École pratique des hautes études (EPHE), il suit les séminaires de Rolf Stein (1911-1999) et de Max Kaltenmark (1910-2002); auditeur unique parfois, il est ensuite rejoint par Anna Seidel (1938-1991). Il y soutient en 1962 le diplôme de l'École (qui ne décernera de diplômes nationaux, dont le doctorat, que bien plus tard), avec un travail sur un texte médiéval, le Han Wudi neizhuan 漢武 帝内傳, qui relate l'initiation mystique du célèbre empereur. Le livre issu du mémoire est publié dès $1965^{3}$.

C' est donc l' histoire de l' art qui a mené Rik aux études taoïstes, et il y reviendra tout au long de sa carrière ${ }^{4}$;' art chinois avait profondément marqué sa sensibilité et son amour de la beauté. À Paris, Rik devint plus parisien qu'un Parisien de naissance; il aimait profondément la ville et sa culture et en partageait la gouaille et l' impertinence. Il demanda et acquit avec fierté la nationalité française, et il ne manquait pas de se fâcher lorsque quelqu' un le désignait comme un «sinologue hollandais», mais c'est dans un environnement éclectique et multilingue qu'il était particulièrement à son aise. Il écrivait et parlait élégamment le français, l' anglais, le néerlandais, le mandarin et le hokkien, ainsi que l'allemand et le japonais sans toutefois rédiger dans ces deux dernières langues. S' exprimer sans grâce était pour lui un signe d'affligeante médiocrité.

Dès l'obtention de son diplôme, Rik est recruté à l'École française d'Extrême-Orient (EFEO) comme chercheur de 1962 à 1973. Il part aussitôt à Taïwan, où l' attend un poste à l'Academia Sinica à Taipei. Le contexte politique et idéologique y est peu propice à l' étude du taoïsme et des savants taïwanais lui disent que cette religion a tout simplement disparu. Mais, après avoir rencontré par hasard une procession, il comprend qu'il n' en est rien. Sa curiosité et son pen-

3 L'empereur Wou des Han dans la légende taoïste (Paris, École française d'Extrême-Orient, 1965).

4 «The True Form: Reflections on the Liturgical Basis of Taoist Art», Sanjiao wenxian 三教文 獻. Matériaux pour l'étude de la religion chinoise 4 (2005): 91-113. 
chant naturel l'éloignent des bibliothèques pour le conduire dans les temples, où il assiste en 1964 à un grand rituel d' offrande jiao 醮 célébré par les taoïstes de l'éminente famille Chen de Tainan. Cette expérience constitue un tournant décisif; il s'installe à Tainan, où il accueillera et initiera à la religion vivante un grand nombre de chercheurs occidentaux. Il est adopté comme frère juré par Chen Yung-sheng 陳榮盛 (1927-2014) et intègre la troupe de sa famille. Rik apprend la liturgie, ainsi que la musique et tous les arts taoïstes, court les villages pour célébrer les rituels fastes (célébration des dieux locaux) et sombres (funérailles), et vit dans l'«autre» Taïwan qui ne parle que hokkien, ce qui lui vaudra des rapports tendus et des rencontres musclées avec les autorités du Kuomintang. Son épouse Wendela et leurs deux filles, nées à Taïwan, sont partie prenante de cette vie intégrée à la société locale. La formation du futur maître taoïste est couronnée en mars 1966 par son ordination, présidée par le $63^{\mathrm{e}}$ Maître céleste Zhang Enpu 張恩溥 (1904-1969).

Tout en étudiant le rituel, Rik se plonge dans les textes et commence à découvrir les corrélations profondes entre les manuscrits de la famille Chen qu'il a recopiés et le Canon taoïste. Depuis sa situation privilégiée d' officiant taoïste, il observe aussi la société locale et les organisations qui invitent les daoshi 道士 à célébrer leurs dieux avec elles, et qu' il appellera par la suite les «structures liturgiques de la société locale ${ }^{5}$. Il se passionne notamment pour le théâtre de marionnettes ${ }^{6}$, dont il montre la fonction rituelle fondamentale, et pour les groupes de musiciens amateurs qui pratiquent ensemble, le soir dans les temples, le nanyin 南音 (ou nanguan 南管); il organisera en 1982 pour la chanteuse Tsai Hsiao-yue 蔡小月 et sa troupe, le Nanshengshe 南聲社, une tournée en France et des enregistrements, les premiers qui rendirent accessibles au public européen cette magnifique tradition ${ }^{7}$.

L'impact de la découverte par Rik de la tradition vivante du taoïsme fut considérable. Un des moments fondateurs fut le colloque de Bellagio, du 7 au 14 septembre 1968, la première rencontre internationale d'études taoïstes, organisée par Holmes Welch (1924-1981) et Anna Seidel. Tandis que les autres participants sont spécialistes des sources textuelles anciennes, Rik décrit la pratique de ces textes dans le rituel. Sa communication, que Rik ne publiera jamais en espérant toujours l' améliorer, figure certainement au palmarès des manuscrits les plus cités.

5 «Neighborhood Cult Associations in Traditional Tainan», in The City in Late Imperial China, éd. G. William Skinner (Stanford, Stanford Univ. Press, 1977), 651-676.

6 «The Divine Jester: Some Remarks on the Gods of the Chinese Marionette Theater», Bulletin of the Institute of Ethnology, Academia Sinica 21 (1966): 81-95.

7 Cette tournée visita aussi quatre autres pays; les enregistrements furent diffusés dans la série Ocora. 
De son travail de terrain, Rik tire des articles fondateurs; ainsi son essai sur la différence et la complémentarité entre les prêtres de la liturgie classique, daoshi, et les exorcistes, fashi 法師 $^{8}$. Il publie un livre sur un petit rite, le partage des lampes, fendeng 分燈, pour servir de modèle d' analyse de la liturgie ${ }^{9}$. Mais son travail de terrain ne sera jamais pleinement publié. Il parlait, encore l'an dernier, de se mettre enfin à l'écriture d'un livre qui donnerait une présentation complète et une analyse globale de son expérience à Taïwan. Cet ouvrage de réflexion ne pourra désormais voir le jour. Il l'a longtemps jugé prématuré pour différentes raisons, parmi lesquelles sans doute l'envie de construire des projets collectifs plutôt que d'écrire seul et le besoin, partagé par nombre de ses disciples et collaborateurs, de comprendre les racines historiques de ce qu'il avait observé dans les années 1960-1970.

De retour de Taïwan en 1972, Rik doit répondre à une autre urgence, celle d'enseigner et de faire comprendre la réalité du taoïsme à un public qui n' avait pas la moindre idée de ce que pouvait être un grand rituel d'offrande jiao de sept jours. Il enseigne à la section des Sciences religieuses de l' E PH E, sur recommandation de Claude Lévi-Strauss, dès 1972 et sera titularisé directeur d'Etudes non cumulant en janvier 1973. De cet enseignement, qui puisait à des sources beaucoup plus larges que son matériau ethnographique et liturgique, il tire sa première grande œuvre, un livre de synthèse et un des très rares best-sellers de la discipline, Le corps taöste, paru en 1982 et rapidement traduit en plusieurs langues ${ }^{10}$. Le corps taoïste a profondément marqué tous les historiens des religions chinoises et bien au-delà, tant le livre est de ceux qu' on ne peut refermer avant de les avoir lus en entier. Il y déploie ce qu'il continuera à développer dans ses travaux ultérieurs: une vision de la culture chinoise dans son ensemble, profondément cohérente et où les pratiques individuelles - nourries par l'aspiration à l' autonomie et à la liberté et dont la plus haute expression est l'immortalité - sont organiquement liées à une vision de la société et à des formes d' organisation locales toujours en tension avec un État central, le tout s'exprimant dans des pratiques rituelles et artistiques.

Son enseignement à l' E P H E dura presque trente ans, de 1973 à 2000. Le séminaire du vendredi après-midi, autour de la longue table en bois massif de la salle

8 «Vernacular and Classical Ritual in Taoism», Journal of Asian Studies 45 (1985): 21-57.

9 Le fen-teng: Rituel taoïste (Paris, Publications de l'École française d'Extrême-Orient 104, 1975).

10 Le corps taoïste (Paris, Fayard, 1982); Il corpo taoista - Corpo fisico, corpo sociale, tr. Fabrizio Pregadio (Rome, Ubaldini, 1983); Tao, de levende religie van China, traduction de l' auteur (Amsterdam, Meulenhoff, 1988); The Taoist Body, tr. Karen C. Duval (Berkeley, Univ. of California Press, 1993); cette liste n' est pas exhaustive. 
Marcel Mauss de l' escalier E de la Sorbonne, est devenu légendaire. S'y pressent étudiants et auditeurs - nombreux, attirés par l' exotisme du sujet puis devenus fidèles après avoir compris que le taoïsme réel était infiniment plus intéressant que celui qu'ils fantasmaient. Viennent aussi des collègues déjà en poste mais ne pouvant se résoudre à se couper de cette source inépuisable d'idées; on y voit encore des collègues étrangers de passage. Il serait difficile de trouver un sinologue spécialiste du taoïsme en Europe et en Amérique du Nord n'ayant pas passé au moins quelques mois à Paris auprès de Rik; toutes et tous ont des histoires à raconter sur la générosité du maître.

L' enseignement de Rik portait sur des sujets extraordinairement variés ${ }^{11}$ et il était toujours vivant, libre et spontané. Rik manquait rarement l'occasion de plaisanter ou de se moquer gentiment des personnes aux certitudes très assises. Dans l'esprit de l'EPHE, il apportait des matériaux et les lisait avec les étudiants et les auditeurs: des manuscrits liturgiques, dont certains de sa propre collection; des textes fondateurs, dont le Zhuangzi; des sources historiques, notamment l' épigraphie qu' il appréciait particulièrement, surtout pour le témoignage direct offert par les inscriptions qui sont en général des sources locales, non réécrites - sauf exception - par le pouvoir central; des matériaux ethnographiques, aussi. Son intérêt de longue date pour la psychanalyse le mena à consacrer quelque temps son séminaire à l'ethnopsychiatrie. Il n'est aucune période qu'il n' ait pas étudiée: l' antiquité à laquelle il revenait toujours; les Han et la période médiévale, avec l'émergence de l'Église du maître céleste 天師道 et des premiers textes liturgiques taoïstes ${ }^{12}$, mais aussi le culte des immortels; les Tang et les Song, avec l'essor des temples locaux et la définition des relations entre les Trois enseignements - qui donna lieu à un article paru dans le T'oung $\mathrm{Pao}^{13}$; les Ming et les Qing, avec notamment les temples de Pékin; les transformations modernes et contemporaines. En écho à ce vaste champ de domaines et de disciplines, il accueillait et formait des étudiants venus d'horizons très variés: histoire, pensée, ethnologie, histoire de l'art. Il aurait sans doute souhaité voir davantage de ses élèves étudier le rituel sur le terrain: «On en apprend plus en une journée sur le terrain qu' en un an en bibliothèque», répétait-il à l' envi. Cependant, il avait aussi une grande pratique

\footnotetext{
11 Les résumés de tous ses cours sont disponibles sur l'annuaire de la Section des Sciences religieuses: https://www.persee.fr/collection/ephe.

12 Il consacra à ce sujet un article de synthèse marquant la fin de son enseignement à l' E PHE : «Le pacte de pureté du taoïsme», Annuaire de l'École pratique des hautes études, Section des Sciences religieuses 109 (2000-2001): 29-53.

13 «Purity and Strangers: Shifting Boundaries in Medieval Taoism», Toung Pao 8o (1994): $61-81$.
} 
des bibliothèques, comme lorsqu' il partit au Japon - où il noua de très solides amitiés - étudier les éditions anciennes du Canon taoïste; il administra avec soin et passion la bibliothèque de l'Institut des Hautes Études Chinoises, dont il fut le directeur de 1988 à 1992, et fonda plus tard sa propre bibliothèque en Chine.

Rik s'engagea aussi pleinement dans les institutions qui participent à coordonner et à défendre les études orientales au niveau français et européen, dans des activités souvent chronophages. Il fut ainsi membre du Conseil de la Société asiatique au poste de trésorier qu' il assuma de 1973 à 1993 et, en 1991, devant la menace d'émiettement des études orientales au sein du CNRS, il utilisa l' outil fédérateur que constitue la Société asiatique pour organiser à l'IMA un vaste colloque intitulé «Pour une nouvelle politique de l'orientalisme» qui associa toutes les composantes de l' orientalisme français et des représentants de divers pays européens qui se liguaient contre les politiques de marginalisation des études orientales. Ce colloque fut suivi de la publication d'un Livre blanc de l'orientalisme français.

Lors du départ à la retraite d'Erik Zürcher (1928-2008), Rik est nommé professeur à l'université de Leiden (1993-1999). Il s'y installe tout en continuant à assurer son séminaire à Paris, désormais le samedi matin. Il trouve à Leiden un cadre pour enseigner non seulement le taoïsme, mais aussi la civilisation chinoise dans son ensemble, en rassemblant, selon ses termes, toutes les pièces du puzzle de la cohérence culturelle du monde chinois. Il y renoue aussi avec le plaisir de parler et d'écrire dans sa langue natale ${ }^{14}$. Il publiera tardivement (mais il s' agit d'un travail commencé longtemps auparavant) des traductions en néerlandais du Daodejing ${ }^{15}$, du Lunyu ${ }^{16}$ et, surtout, du Zhuangzi ${ }^{17}$. Le Zhuangzi l'a accompagné toute sa vie, il n' avait de cesse d'y revenir et d'y trouver toujours davantage de preuves de l'absurdité de séparer ce texte de la tradition religieuse vivante.

Rik adorait enseigner, et il donnait encore un séminaire informel pour les étudiants à Paris quand la pandémie de Covid-19 y mit fin en mars 2020; mais il aimait tout autant la recherche collective. Sa carrière est marquée par une

14 Voir le volume d' hommage compilé par ses collègues de Leiden: Linked Faiths: Essays on Chinese Religions and Traditional Culture in Honour of Kristofer Schipper, éd. Jan A.M. de Meyer and Peter M. Engelfriet (Leiden, Brill, 200o).

15 Lao Zi: Het boekvan de Tao en de innerlijke kracht (Amsterdam, Uitgeverij Augustus, 2010).

16 Confucius. De gesprekken. Gevolgd door Het leven van Confucius door Sima Qian (ca. 145-86 v. Chr.) (Amsterdam, Uitgeverij Augustus, 2014).

17 Zhuang Zi:De volledige geschriften, het grote klassieke boek van het taö̈sme (Amsterdam, Uitgeverij Augustus, 2014); une version antérieure, avec seulement les chapitres intérieurs, était parue en 1997 . 
succession de grands projets dont il était l'âme et l' animateur infatigable. Le premier d'entre eux fut le catalogue raisonné du Canon taoïste (Daozang 道 藏). Dernier grand canon des traditions religieuses mondiales à ne pas être doté d'un outil de travail systématique, le Daozang n' avait alors, dans les années 1970, même pas d'édition ponctuée. Rik rassembla une équipe pour décrire et analyser ses quelque mille cinq cents ouvrages; elle était localisée dans le chaleureux foyer du Centre de documentation et d'étude du taoïsme, dans un bureau de la Maison de l'Asie, avenue du Président Wilson, à deux pas du Musée Guimet.

L' entreprise est novatrice à de nombreux égards. Elle l' est par sa structure, d' abord, avec un contrat de la Fondation Européenne de la Science (1978-1984), qui en fait un des premiers projets sinologiques à être reconnu par ce type de grand financement. Elle innove aussi par la création d'une vraie communauté scientifique internationale; des jeunes chercheurs viennent participer au projet de France, d'Allemagne, d'Italie, du Royaume-Uni, et bientôt des États-Unis, avec John Lagerwey qui rejoint l' équipe après avoir soutenu sa thèse à Harvard: tous publieront des ouvrages et des articles issus de leur travail au sein du projet. Elle innove encore par son usage de la technologie: les noms, dates, lieux, titres et autres informations identifiées dans chaque texte sont systématiquement saisis sur ordinateur, triés et imprimés; j' utilisais encore ces index dans les années 1990, à une époque qui doit sembler lointaine à la jeune génération habituée à trouver les textes en ligne ${ }^{18}$. C' est sur la base de ces index que les contributeurs ont pu avancer dans la datation des textes, l'identification des filiations textuelles et des emprunts implicites ou explicites, le regroupement des écoles, bref, créer de l' ordre dans la masse documentaire écrasante du Dao$z a n g$. Le projet ralentit après 1984 avec la fin du financement et l' évolution des carrières de bon nombre de ses membres; la majorité des notices sont écrites mais il reste du travail. Franciscus Verellen rejoint Rik quelques années plus tard et ils reprennent le manuscrit, rédigeant les notices manquantes, corrigeant et mettant à jour celles déjà existantes, et construisant l'architecture d'ensemble: l' œuvre est publiée en $2004^{19}$.

18 Rik avait déjà, dès ses années taïwanaises, compilé à la main et publié des index préparatoires sur des textes essentiels tels le Baopuzi 抱朴子 et le Huangtingjing 黃庭經, puis le Yunji qiqian 雲笈七籤: Concordance du Pao-p'ou-tseu nei-p'ien et Concordance du Pao-p'ou-tseu wai-p'ien (Paris, E FEO, 1965-1969); Concordance du Houang-t'ing king (Paris, E FEO, 1975); Projet Tao-tsang: Index du Yunji qiqian (Paris, E FEO, 1981-1982, 2 vols.).

19 The Taoist Canon: A Historical Companion to the Daozang - Daozang Tongkao 道藏通考, éd. Kristofer Schipper and Franciscus Verellen (Chicago, Univ. of Chicago Press, 2004, 3 vols.). 
Le grand projet suivant sera «Pékin, ville sainte». Très tôt, dès l'ouverture progressive de la Chine populaire après 1978, Rik est attiré par l'immense champ d' investigation que constitue la Chine continentale. Il est persuadé que la vitalité actuelle du taoïsme, qu'il a démontrée dans le cas de Taïwan, existe aussi de l'autre côté du détroit. Il demeure sensible aux remarques parfois critiques de collègues qui affirment que ses théories ne sont pas valables pour le monde chinois en général, mais seulement pour le cas très particulier de Taïwan. Or Rik est un ardent défenseur de l' autonomie et de la spécificité des sociétés locales, mais aussi de la cohérence culturelle du monde chinois. Il se propose donc de montrer que ce qui vaut pour Tainan, à savoir que la société y est organisée en communautés autonomes de culte à leurs saints avec le rituel taoïste pour leur conférer structure et légitimité, se révélera tout aussi vrai sous les murs mêmes de la Cité interdite. Il lance ce projet en 1995 et c' est une nouvelle génération de chercheurs qui s'y forme, y compris l' auteur de ces lignes.

Le projet «Pékin ville sainte» comprend des enquêtes de terrain, bien sûr, avec de mémorables visites en groupe et de nombreuses missions individuelles. Il consacre une grande partie de ses efforts à la lecture collective des inscriptions: la publication alors toute récente de cent volumes d'estampages conservés à la Bibliothèque nationale de Pékin et qui incluent le résultat de campagnes systématiques de relevés des stèles des temples de Pékin lui en fournit le matériau. Rik fonde une revue pour publier ces matériaux: Sanjiao wenxian 三教文獻. Matériaux pour l'étude de la religion chinoise: on n'y publiera pas de grandes analyses ni de théorie, affirme-t-il, le temps viendra plus tard; pour le moment il faut, selon la formule qu' il utilisait si souvent, «dire non pas le dernier mot, mais le premier» et présenter des matériaux inédits ${ }^{20}$. Le premier numéro s' ouvre cependant sur un article programmatique, dans lequel il développe la notion de structures liturgiques ${ }^{21}$.

Parmi tous les temples de Pékin, l' un retient particulièrement son attention, et dans l' article susnommé il en raconte la découverte émue depuis la fenêtre d'une tour d'habitation voisine: le temple du Pic de l'Est, Dongyuemiao 東获 廟22. Ce temple représentait un modèle de ce qu'était pour lui la société civile chinoise: une fondation officielle, patronnée par l'État impérial, mais gérée par des taoïstes et abritant des centaines d'associations pieuses et de guildes qui

$20 \quad$ Quatre numéros ont paru, de 1997 à 2004.

21 《Structures liturgiques et société civile à Pékin», Sanjiao wenxian 三教文獻. Matériaux pour l'étude de la religion chinoise 1 (1997): 9-23.

22 «Note sur l'histoire du Dongyue miao de Pékin», Hommage à Kwong Hing Foon. Études d'histoire culturelle de la Chine, éd. Jean-Pierre Diény (Paris, Collège de France, Institut des Hautes Études Chinoises, 1995), 255-269. 
en finançaient les activités rituelles, philanthropiques et artistiques. Comme pour le projet Daozang, «Pékin, ville sainte» fut une pépinière de vocations et d'idées. Il s'est interrompu en 2001 faute de financement et de soutien institutionnel, mais a été repris dès 2002 par Marianne Bujard qui l'a mené sans discontinuer et a déjà publié cinq gros volumes de matériaux sur les onze qui sont prévus ${ }^{23}$.

Sa retraite à l'EPHE en 2000 et à Leiden en 1999 laisse Rik libre d' aller créer ailleurs de nouveaux projets. Avec sa seconde épouse, la professeure Yuan Bingling, il s' installe à Fuzhou où il est nommé professeur à Fuzhou daxue. Il y fonde une bibliothèque, le Xiguan cangshulou 西觀藏書樓, le Belvédère des livres du Pavillon occidental, dont le nom évoque les racines taoïstes de la grande bibliothèque impériale des Han. Il lui donne une partie importante de sa bibliothèque personnelle et acquiert, grâce à des donations qu'il a su solliciter, des collections de livres en langues occidentales (anglais, français, allemand...) concernant les sciences humaines ainsi que la littérature et les lettres classiques afin de faciliter les échanges culturels et intellectuels entre la Chine et l'Europe. Sa maison à Fuzhou, comme celles, auparavant, à Tainan et à Paris, est grande ouverte aux amis, aux collègues et aux étudiants. Rik enseigne en Chine, à Fuzhou et à Pékin - ses cours à l'Université de Pékin dans les années 2010 remplissent les amphithéâtres -, il est distingué par des prix et des honneurs comme, en 2004, la médaille de l' amitié 中國人民共和國友誼獎. $S$ 'insérer dans le monde académique, politique et médiatique de la Chine du $\mathrm{XXI}^{\mathrm{e}}$ siècle est pour lui un nouveau terrain, et il répète à l'envi qu' il y apprend autant que, trente ans auparavant, dans les villages des environs de Tainan. Il lance à Pékin un grand projet de traduction en anglais des Classiques; il n'a jamais perdu de vue au cours de sa carrière les Sishu wujing 四書五經 et la question de la continuité culturelle entre l' antiquité et la société contemporaine, et il y consacre pendant ces années 2000 toute son attention, tant il est persuadé que la compréhension de la tradition vivante permet de relire et d'aborder autrement les textes fondateurs et la nature du grand tournant intellectuel du $\mathrm{IV}^{\mathrm{e}}$ siècle avant notre ère, et de réécrire radicalement l' histoire chinoise. Le projet de traduction rencontre des obstacles qui ne sont pas d'ordre scientifique, mais sa réflexion, collective et individuelle, se poursuit.

Le dernier grand projet de Rik est celui des Sanctuaires de la nature, pour lequel il crée une fondation, Aishan 愛山, avec Yuan Bingling ${ }^{24}$. Intéressé de longue date par les rapports entre taoïsme et écologie, il élabore ce projet visant

\footnotetext{
23 Beijing neicheng simiao beike zhi 北京內城寺廟碑刻志, éd. Marianne Bujard (Beijing, Guojia tushuguan chubanshe, 2011-).

24 https://www.aishanfoundation.org/.
} 
à documenter par les textes et par l'ethnographie non seulement les Trentesix «grottes-cieux», dongtian 洞天 et les Soixante-douze «terres bénies», fudi 福地, dont la liste fut codifiée sous les Tang, mais aussi, au-delà de cette géographie sacrée taoïste déjà dense, les innombrables lieux saints que l'on trouve dans chaque recoin du monde chinois et dont l'histoire est souvent très ancienne. Il y voyait des réservoirs de biodiversité, grâce aux interdits de chasse, de pêche et de cueillette et aux pratiques de préservation. Il espérait ainsi montrer le lien très intime entre l'histoire des rites et des cultes des saints locaux et l'histoire de l' environnement; il rêvait de trouver des financements pour permettre à des étudiants et des jeunes chercheurs de consacrer des thèses et des travaux à chacun de ces sites. Il avait lui-même fait ce travail pour un lieu saint au nord de Fuzhou, crucial pour l'histoire du taoïsme médiéval, le Huotongshan 霍童山 ${ }^{25}$.

L' œuvre de Rik est immense et variée, traversée de bout en bout par un certain nombre de thèmes forts. Je voudrais ici en évoquer deux. Le premier est son attachement à ce qu' il appelait «l' autre Chine » ou le «pays réel», celui des langues et des sociétés locales, dont il voulait montrer le caractère communautaire, voire démocratique, en tension permanente avec les forces centripètes du pouvoir impérial et de ses successeurs. Il considérait la vision taoïste du monde comme un outil puissant qui permettait d' intégrer en un tout cohérent la multiplicité des sociétés locales sans écraser leur autonomie et leurs spécificités. Cette conception était nourrie par l'humanisme inspiré de sa propre expérience de vie; il était confiant dans la capacité des sociétés locales à perdurer au XXI ${ }^{\mathrm{e}}$ siècle. Elle sous-tendait aussi sa conviction de la nécessité intellectuelle de mettre (ou remettre) le taoïsme au centre des études chinoises.

Le second thème transversal de son œuvre est «la tradition vivante», dont il fit le sous-titre d' une collection d'essais et d' articles parue en ${ }^{2008^{26}}$. Il signifiait ainsi que rien de l'histoire culturelle et religieuse de la Chine n'a totalement et définitivement disparu, et que l'on peut retrouver, sous forme de bribes, parfois difficilement reconnaissables, des formes vivantes, pratiquées aujourd'hui, des rites, des arts, des cultes et des formes d'organisation sociale qui ont structurél'évolution des sociétés chinoises depuis l' antiquité. Une telle conviction le portait aisément à proposer de grandes hypothèses et des schémas d'évolution historique dans la très longue durée ${ }^{27}$, qui ne recevaient pas

25 《Diyi dongtian: Mindong Ningde Huotongshan chukao» 第一洞天 : 閩東甯德霍童 山初考, Zhongguo wenhua jiyinku 中國文化基因庫 (Beijing, Beijing daxue chubanshe, 2002).

26 La religion de la Chine. La tradition vivante (Paris, Fayard, 2008).

27 «Taoism: The Story of the Way», in Taoism and the Arts of China, éd. Stephen Little with 
toujours l' adhésion de sinologues demandant des preuves de ce qu' il avançait. Rik proposait toujours ces grands schémas comme des postulats devant nous mener à découvrir de nouvelles sources ou à relire d' un œil nouveau les textes connus. De telles hypothèses pouvaient, bien sûr, se révéler à l'étude devoir être radicalement revues, ce qu' il acceptait de très bon cœur, mais le simple fait de les avoir explorées ouvrait des champs de connaissance entièrement nouveaux. J' ai eu le bonheur de faire partie de celles et ceux, nombreux, qui ont vécu cette expérience.

Shawn Eichman (Chicago, The Art Institute of Chicago, in association with Univ. of California Press, 2000), 33-55. 